\title{
Rheological behavior of heated starch dispersions in excess water: role of starch granule
}

\author{
M.A. Rao, ${ }^{a}$ P. E. Okechukwu, ${ }^{a}$ P.M.S. Da Silva ${ }^{b}$ and J.C. Oliveira ${ }^{b}$ \\ ${ }^{a}$ Department of Food Science and Technology, Cornell University Geneva, New York, New York 14456-0462, USA \\ ${ }^{b}$ Escola Superior de Biotecnologia, U. Catolica Portuguesa, R. Dr. Antonio Bernardino de Almeida, 4200 Porto, Portugal
}

\begin{abstract}
Granule size and size distribution, measured by laser diffraction, affected the flow behavior at $20^{\circ} \mathrm{C}$ of $(2.6 \% \mathrm{w} / \mathrm{w})$ corn and cowpea starch dispersions heated for various time intervals above their gelatinization temperatures. The standard deviation of the granules' size described the transition of flow behavior from shear thickening in the early stages of gelatinization to shear thinning in the latter stages and influenced the critical shear rate, $\dot{\gamma}_{c}$, for the onset of shear thickening in starch dispersions. The granules swelled to a maximum of about 3.5 times the raw starch granule mean diameter and $65 \%$ granule mass fraction. The consistency index of the dispersions increased with granule mean diameter Modified waxy maize starch dispersions heated at $80^{\circ} \mathrm{C}$ exhibited antithixotropic behavior at a shear rate of $200 \mathrm{~s}^{-1}$; both dynamic frequency data and Cox-Merz plots revealed their gel-like behavior. Copyright (C) 1997 Elsevier Science Ltd. All rights reserved
\end{abstract}

\begin{tabular}{|c|c|}
\hline \multicolumn{2}{|c|}{ NOMENCLATURE } \\
\hline$A, B$ & constants in eqn (9) \\
\hline & starch concentration, w/w \\
\hline$c Q$ & starch granule mass fraction \\
\hline$d_{\max }$ & maximum mean diameter ratio \\
\hline$D$ & starch granule diameter at any time \\
\hline $\begin{array}{l}D_{0} \\
G^{\prime}\end{array}$ & $\begin{array}{l}\text { initial average starch granule diameter } \\
\text { storage modulus }(\mathrm{Pa})\end{array}$ \\
\hline$G^{\prime \prime}$ & loss modulus $(\mathrm{Pa})$ \\
\hline$G_{0}$ & $\begin{array}{l}\text { plateau storage modulus, } G^{\prime} \text { value at high } \\
\text { frequencies, }(\mathrm{Pa})\end{array}$ \\
\hline$G_{\mathrm{p}}$ & $\begin{array}{l}\text { shear modulus of material of spherical } \\
\text { particle (in eqn (7)) }\end{array}$ \\
\hline GSID & starch granule size distribution \\
\hline$K$ & consistency index $\left(\mathrm{Pa} \mathrm{s}^{n}\right)$ \\
\hline$K_{\mathrm{o}}$ & constant in eqn (7) (Pa s$\left.)^{n}\right)$ \\
\hline$n$ & flow behavior index (dimensionless) \\
\hline$Q$ & $\begin{array}{l}\text { mass of heated starch granules per unit } \\
\text { weight of dry starch }\end{array}$ \\
\hline $\operatorname{Re}$ & particle Reynolds number, $=\frac{D^{2} ; \rho}{\eta}$ \\
\hline SD & $\begin{array}{l}\text { standard deviation of gelatinized starch } \\
\text { granules }\end{array}$ \\
\hline $\mathrm{SD}_{\mathrm{o}}$ & $\begin{array}{l}\text { standard deviation of ungelatinized starch } \\
\text { granules }\end{array}$ \\
\hline & starch granule mass fraction \\
\hline
\end{tabular}

\section{NOMENCLATURE}

$c Q \quad$ starch granule mass fraction

$d_{\max }$

$D$

$D_{0}$

$G^{\prime}$

$G^{\prime \prime}$

$G_{\mathrm{p}}$

GSID

$K$

$K_{\mathrm{o}}$

starch granule diameter at any time

initial average starch granule diameter

storage modulus $(\mathrm{Pa})$

plateau storage modulus, $G^{\prime}$ value at high

frequencies, $(\mathrm{Pa})$

particle (in eqn (7))

consistency index $\left(\mathrm{Pa} \mathrm{s}^{n}\right)$

constant in eqn (7) (Pa $\left.\mathrm{s}^{n}\right)$

wehavior index (dimensionless)

weight of dry starch

weight of dry starch

granules

granules

starch granule mass fraction

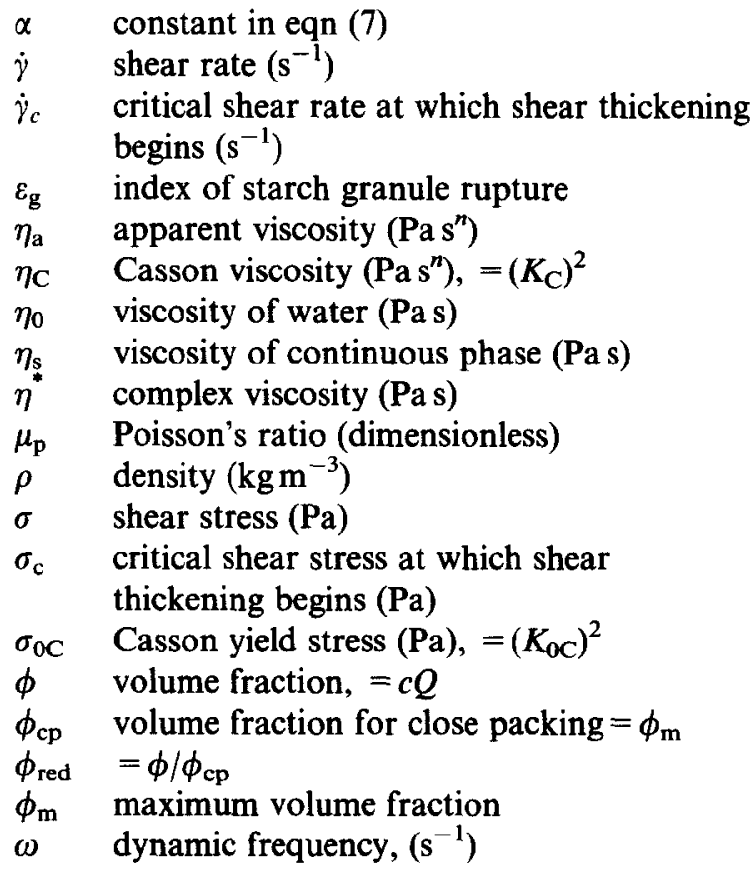




\section{INTRODUCTION}

Starch is a major source of calories and raw material in food and process industries. In foods, one major use of starch is as a thickening/gelling agent. The swelling behavior of starch granules is hindered at high starch (limited moisture) concentrations, but not at low starch (excess moisture) concentrations (Liu and Lelievre, 1993). In this study, we focus on starch granules heated under excess moisture conditions where gelatinization follows pseudo-first-order-Arrhenius kinetics (Lund, 1984; Kokini et al., 1992). One may also view gelatinized starch dispersions as microgel systems (Ketz et al., 1988; Evans and Lips, 1993) whose flow and viscoelastic behavior are strongly influenced by the physical state of the granules (size and size distribution) (Evans and Lips, 1992; Okechukwu and Rao, 1995). It should be possible to use starch granules of selected sizes and size distributions as ingredients to obtain different rheological characteristics in new food formulations.

Gelatinized starch dispersions are non-Newtonian fluids that may also exhibit yield stress at low shear rates (Evans and Haisman, 1979; Christianson and Bagley, 1984; Giboreau et al., 1994). Most studies in rheology and process applications have dealt with shear thinning (pseudoplastic) foods, but shear thickening (dilatant) rheological behavior is also of interest as an area of study in food rheology because it has been observed (Bagley and Christianson, 1982; Dail and Steffe, 1990a; Dintzis and Bagley, 1995 Okechukwu and Rao, 1995) and its potential use needs to be explored. In particular, the conditions under which shear thickening rheological behavior occurs in gelatinized (partially or completely) starch dispersions would be of interest in food processing (e.g. thermal processing and holding tubes) and product applications.

Hoffmann's study (Hoffmann, 1972) on polyvinyl chloride spheres in dioctyl phthalate dispersions pointed out that shear thickening flow behavior is a reproducible phenomenon. Hoffmann also suggested that: 'The discontinuous viscosity behavior which occurs at solids concentrations above 0.50 is caused by a flow instability in which surfaces of spheres, packed in two dimensional hexagonal packing at low shear rates, break up into less ordered arrays of spheres. In any given viscometric flow the ordered surfaces of spheres are parallel to surfaces of constant shear and one axis of the packing always points in the direction of flow'. As shear rate is increased, the loss of longrange orientational order that results in discontinuous viscosity behavior is also referred to as 'shear melting' (Chow and Zukoski, 1995b). Particle size and distribution, particle volume fraction, particle shape, particle-particle interaction, continuous phase viscosity, and type, rate and time of deformation are important factors in shear thickening (Barnes, 1989; Boersma et al., 1990, 1991; Laun et al., 1991).
Following Einstein's work (Einstein, 1906) on dilute rigid sphere dispersions, models for estimating viscosity of concentrated non-food dispersions of solids are based on the volume fraction $(\phi)$ of the suspended granules and the viscosity of the continuous phase (Jinescu, 1974; Metzner, 1985). Because of the deformable nature of gelatinized starch granules, it is difficult to determine $\phi$ accurately. Instead of $\phi$, it is preferable to work with the starch granule mass (SGM) fraction, $c Q$, where $c$ is starch concentration, $\mathrm{w} / \mathrm{w}$, and $Q$ is mass of swollen starch granules in excess water per unit weight of dry starch. The SGM fraction is unique in that it takes into consideration both solids concentration and volume of starch granules after water absorption, and it was shown to play an important role in power law consistency and the yield stress of starch dispersions (Bagley and Christianson, 1982; Okechukwu and Rao, 1995).

A number of studies have shown that dispersions of starches from various botanical sources, for the most part, exhibit pseudoplastic flow behavior, and that SGM fraction plays an important role (Evans and Haisman, 1979; Ellis et al., 1989; Noel et al., 1993). SGM has been referred to as 'notional volume fraction' (Evans and Lips, 1992). However, determination of SGM fraction (centrifugation, careful separation of granules, and weighing the wet granules) requires considerable care and labor, and the data are prone to considerable scatter. Therefore, it would be desirable to replace the current SGM method with another method that is less laborious and, preferably, automated.

Although shear thickening behavior of non-food colloidal dispersions, usually of $\mathrm{TiO}_{2}$ particles, has been studied, only recently has the role of particle morphology been examined (D'Haene et al., 1993). Studies on rigid particles with diameters $1-600 \mathrm{~nm}$ point out several important features of shear thickening (Barnes, 1989; Boersma et al., 1990, 1991; Laun et al., 1991; Chow and Zukoski, 1995a, b). However, the smaller diameter particles and higher viscosity suspending fluids, such as dioctyl phthalate, result in very low particle Reynolds numbers $\left(R e=D^{2} \dot{\gamma} \rho / \eta\right)$ (Russel et al., 1989). To illustrate the order of magnitude, at a fixed $\dot{\gamma}=100 \mathrm{~s}^{-1}$ the particle Reynolds number for typical $500 \mathrm{~nm}$ particles in dioctyl phthalate $\left(\rho=980 \mathrm{~kg} \mathrm{~m}^{-3}, \eta=0.054 \mathrm{Pas}\right)$ is $4.54 \times 10^{-7}$, while that for typical $30 \mu \mathrm{m}$ starch granules in water $\left(\rho=1000 \mathrm{~kg} \mathrm{~m}^{-3}, \quad \eta=0.001 \mathrm{Pas}\right)$ at the same $\dot{\gamma}$ is $9.0 \times 10^{2}$, so that the flow regimes are substantially different. One consequence of shear thickening is that holding tube lengths of food sterilizers will be longer than in the traditional design based on Newtonian flow (Dail and Steffe, 1990a).

Since granules in gelatinized starch dispersions have a size distribution, the rheological behavior of the dispersions depends on their size, size distribution and shape, as well as interaction between the granules, the 
continuous phase viscosity, and the rate and time of deformation (Barnes, 1989; Boersma et al., 1990, 1991; Laun et al., 1991). Changes in granule shape and size in the course of starch gelatinization have been studied using scanning electron microscopy and laser diffraction (Rockland et al., 1977; Galliard and Bowler, 1987; Svegmark and Hermansson, 1992; Ziegler et al., 1993; Okechukwu and Rao, 1995). The influence of size and size distribution of heated starch granules on rheological behavior of the dispersions determined in recent studies is summarized here.

\section{EXPERIMENTAL}

Heated dispersions $(2.6 \% \mathrm{w} / \mathrm{w})$ of corn starch (amylose $\sim 20 \%$, Sigma), cowpea $(2.6 \% \mathrm{w} / \mathrm{w}$ ) (amylose $\sim 30 \%$ ), and modified (cross-linked) waxy maize starch (MWM) (National Starch, COL FLO 67, lot TBK 3886) were studied. Cowpea (Vigna unguiculata) starch was extracted as described in Schoch and Maywald (1968) and Cheng-yi and Shin-ming (1981). The recovered prime starch was washed and vacuum dried at $35^{\circ} \mathrm{C}$ for about $48 \mathrm{~h}$ (Okechukwu and Rao, 1996).

A jacketed stainless steel (JSS) vessel $(\approx 350 \mathrm{ml})$ and a water bath (Isotemp, 901, Fisher Scientific, Pittsburgh) were used to maintain the starch dispersions at selected heating temperatures (Okechukwu and Rao, 1995). The corn and cow pea starch dispersions were heated at specific temperatures $\left(67-85^{\circ} \mathrm{C}\right)$ for different lengths of time $(0.5-420 \mathrm{~min})$, while the MWM starch dispersions were heated at $80^{\circ} \mathrm{C}$ for $0.5-30$ min under mild agitation.

\section{Rheological measurements}

Steady shear (shear stress and shear rate) data were obtained on the heated corn starch samples using a stainless steel $4 \mathrm{~cm}$ diameter, $2^{\circ}$ angle cone and plate arrangement of an automated Carri-Med CSL 100 rheometer (TA Instruments, New Castle, DE). With the cowpea starch dispersions, a $6 \mathrm{~cm}$ diameter anodized aluminum cone, $2^{\circ}$ angle, and an upgraded Carri-Med $\mathrm{CSL}^{2} 100$ rheometer were used. Each sample was sheared at $20^{\circ} \mathrm{C}$ continuously from $0.05 \mathrm{~s}^{-1}$ to an upper limit which varied with sample consistency for $300 \mathrm{~s}$, and subsequently back to the initial shear rate again in $300 \mathrm{~s}$. Data taken during the descending segment of the shear cycle were used for flow characterization and estimates of the power law coefficients.

Time-dependent flow and dynamic rheological data were obtained on the MWM starch dispersions with the $6 \mathrm{~cm}$ diameter, $2^{\circ}$ angle aluminum cone; the former were obtained at fixed values of shear rates $\left(100 \mathrm{~s}^{-1}\right.$, $200 \mathrm{~s}^{-1}, 300 \mathrm{~s}^{-1}$, and $400 \mathrm{~s}^{-1}$ ) at $20^{\circ} \mathrm{C}$ for a period of $1200 \mathrm{~s}$, and the latter were obtained in the linear viscoelastic range over the frequency range 0.62-
$62.83 \mathrm{rad} \mathrm{s}^{-1}$. In each experiment, a fresh sample was used and after loading it was allowed to rest for $900 \mathrm{~s}$ to restore structures that might have been disturbed.

\section{Granule size scan}

The size distribution of granules in each starch dispersion was determined with an automated laser diffraction particle size analyzer (LS130, Coulter Corporation, Hialeah, FL). Standard size spherical particles purchased from the National Institute of Standards and Technology were used to verify the integrity of the LS130 predictions. The optical module with a mounted sample vessel was operated at pump speed 51 and polarization intensity differential scattering (PIDS) at 45-50, to generate results with Coulter software 1.53 and the Fraunhofer optical model. The results provided information on the granule size distribution (GSID): granule mean diameter, standard deviation and the skewness and kurtosis of the distribution.

\section{Starch granule mass fraction}

The mass of solids in a $20 \mathrm{ml}$ starch dispersion was determined by centrifugation for $15 \mathrm{~min}$ at $1000 \mathrm{~g}$ (Leach et al., 1959; Eliasson, 1986) and the clear supernatant was removed leaving behind the swollen sediment. The mass of this sediment was determined and used to calculate the parameter $Q$, i.e. the weight of swollen starch granules divided by the dry weight of starch prior to cooking (Bagley and Christianson, 1982). At the low solids concentration of starch used $(2.6 \%)$, it was assumed that the clear supernatant had the same density as water.

\section{RESULTS AND DISCUSSION}

\section{Granule size distribution}

Typical GSIDs of corn starch, cowpea, and the MWM starch are shown in Figs 1-3. The mean diameters and

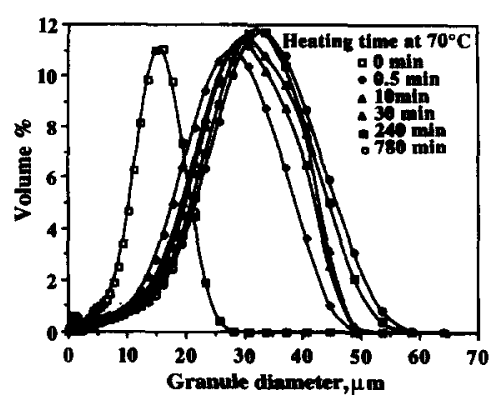

Fig. 1. Size distribution of corn starch granules heated at $70^{\circ} \mathrm{C}$. Note the gradual increase in mean diameter and spread in size distribution with increase in heating time. 


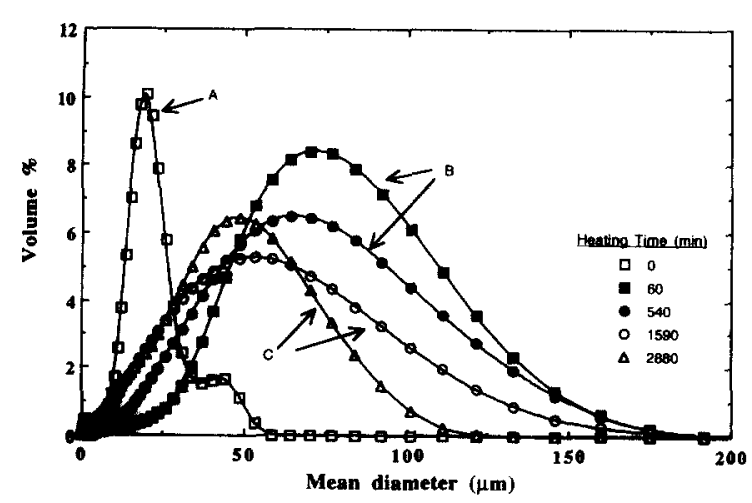

Fig. 2. Granule size distribution of cowpea starch dispersions heated at $80^{\circ} \mathrm{C}$ : (A) raw hydrated granules, (B) fully swollen granules, (C) ruptured granules.

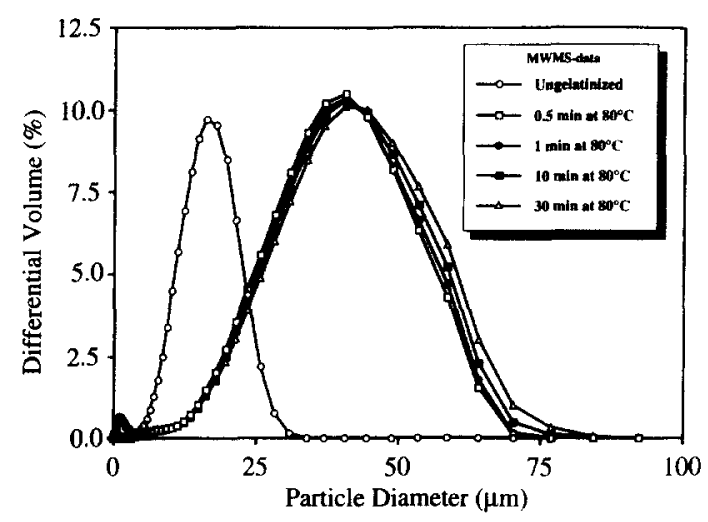

Fig. 3. Size distribution of modified waxy maize starch granules heated at $80^{\circ} \mathrm{C}$. Note that there was a significant increase in mean diameter due to gelatinization, but heating for longer times did not result in significant increase in mean diameter of the granules.

standard deviations of the granules heated at $80^{\circ} \mathrm{C}$ for different times and the corresponding per cent increase with respect to the unheated values are given in Table 1. Among the studied starches, the initial average diameters $\left(D_{0}\right)$ of the granules ranged from $13.5 \mu \mathrm{m}$ for corn starch to $18.7 \mu \mathrm{m}$ for cowpea starch, and the corresponding standard deviations were 6.10 and $8.06 \mu \mathrm{m}$, respectively; after the dispersions were heated for $30 \mathrm{~min}$ at $80^{\circ} \mathrm{C}$, the corresponding diameters were $32.8 \mu \mathrm{m}$ and $67.0 \mu \mathrm{m}$ and the standard deviations were $11.8 \mu \mathrm{m}$ and $28.6 \mu \mathrm{m}$, respectively.

\section{Flow behavior of heated dispersions}

The variation of viscosity with shear rate of corn starch dispersions heated at $70^{\circ} \mathrm{C}$ up to $210 \mathrm{~min}$ revealed (Fig. 4) shear thickening behavior, i.e. the viscosity increased with shear rate. Shear thickening was also observed in samples heated at $75^{\circ} \mathrm{C}$ for $120 \mathrm{~min}$ and at $80^{\circ} \mathrm{C}$ for $5 \mathrm{mins}$ but not in dispersions held at 85 and $90^{\circ} \mathrm{C}$. Figure 5 shows the shear thinning behavior of corn starch when heated at $85^{\circ} \mathrm{C}$, i.e. the viscosity decreased with increasing shear rate. Thus, corn starch dispersion exhibited shear thickening and shear thinning flow behavior at various stages of granule swelling. Shear thickening was observed by Bagley and Christianson (1982) over a shear range of $3-90 \mathrm{~s}^{-1}$ in a $19 \%$ dispersion of wheat starch heated at $60^{\circ} \mathrm{C}$ for $15 \mathrm{~min}$ and characterized at $25^{\circ} \mathrm{C}$; when the dispersion viscosity was measured at $60^{\circ} \mathrm{C}$, the shear thickening shear range shifted to $30-500 \mathrm{~s}^{-1}$. Dail and Steffe (1990a) observed shear thickening in 1.82 and $2.72 \%$ cross-linked waxy maize starch dispersion in a tube $(1.27 \mathrm{~cm})$ viscometer at $121-143^{\circ} \mathrm{C}$ over the shear rate range $40-165 \mathrm{~s}^{-1}$. Dintzis and Bagley (1995) reported shear thickening behavior in Amioca $(98 \%$ amylopectin) $(7.7 \%)$ dispersions heated in cans at $140^{\circ} \mathrm{C}$ and rheological behavior examined at $50^{\circ} \mathrm{C}$ in a $2.61 \mathrm{~mm}$ gap concentric cylinder viscometer.

The power law model (eqn (1)) satisfactorily described the flow behavior of the heated corn and cowpea starch dispersions (Okechukwu and Rao, 1995) in agreement with earlier studies (Wong and Lelievre, 1982; Harrod, 1989).

$$
\sigma=K \dot{\gamma}^{n}
$$

where, $\dot{\gamma}$ is the shear rate in $\mathrm{s}^{-1}, \sigma$ is the shear stress in $\mathrm{Pa}, K$ is the consistency index in $\mathrm{Pa} \mathrm{s}^{\mathrm{n}}$, and $n$ is the flow behavior index (dimensionless). The viscosity of corn starch dispersions rose as the shear rate approached zero, a behavior suggestive of the existence of yield stress (Evans and Haisman, 1979; Doublier, 1981; Wong and Lelievre, 1982; Christianson and Bagley, 1984; Colas, 1986; Giboreau et al., 1994). However, due to the low value of the yield stress, the data fit very well the power law flow model.

Cowpea starch dispersion, held for various times at temperatures above the gelatinization onset, also showed an increase in apparent viscosity as the shear rate approached zero (Fig. 6). At high shear rates, viscosity increased or decreased with shear rate depending on the temperature and time of heating of the dispersion. Shear thinning behavior characterized by values of $n<1.0$ was exhibited by dispersions held at $70-80^{\circ} \mathrm{C}$ for periods $>1 \mathrm{~min}$. Values of $n>1.0$, characteristic of shear thickening dispersions, were oblained for samples held at $67^{\circ} \mathrm{C}$ for heating times up to $6 \mathrm{~h}$.

\section{Critical shear rates}

Given the right circumstances, a dispersion of solid particles may exhibit shear thickening when sheared over a wide range of shear rates (Barnes, 1989). Furthermore, the general apparent viscosity-shear-rate pattern consists of high values of apparent viscosity at low shear rates, immediately followed by shear thinning behavior, and finally an increase in viscosity that begins at a critical shear ratc (Fig. 6). The critical 
Table 1. Average starch granule diameter $(D)$, standard deviation of size distribution $(\sigma)$, and change in average diameter $(\Delta D)$ and standard deviation $(\Delta \sigma)$ due to heating at $80^{\circ} \mathrm{C}$

\begin{tabular}{|c|c|c|c|c|}
\hline Time of heating (min) & $D(\mu \mathrm{m})$ & $\sigma(\mu \mathrm{m})$ & $\Delta D(\%)^{\mathrm{a}}$ & $\Delta D(\%)^{\mathrm{b}}$ \\
\hline \multicolumn{5}{|l|}{ Corn starch } \\
\hline 0.0 & 13.5 & $6 \cdot 1$ & NA & NA \\
\hline $2 \cdot 0$ & 30.6 & 10.9 & 127 & 80 \\
\hline $5 \cdot 0$ & $31 \cdot 3$ & $11 \cdot 1$ & 133 & 84 \\
\hline 30.0 & 32.8 & 11.8 & 144 & 95 \\
\hline $60 \cdot 0$ & $33 \cdot 1$ & 11.9 & 146 & 97 \\
\hline $120 \cdot 0$ & 34.4 & $12 \cdot 1$ & 155 & 100 \\
\hline $300 \cdot 0$ & 34.7 & $12 \cdot 5$ & 157 & 107 \\
\hline $420 \cdot 0$ & 34.9 & $12 \cdot 6$ & 159 & 108 \\
\hline \multicolumn{5}{|l|}{ Cowpea starch } \\
\hline 0.0 & $18 \cdot 7$ & $8 \cdot 1$ & NA & NA \\
\hline 0.5 & 62.6 & 25.8 & 235 & 220 \\
\hline 1.5 & $63 \cdot 6$ & $26 \cdot 5$ & 240 & 229 \\
\hline 3.0 & $66 \cdot 0$ & $27 \cdot 6$ & 253 & 242 \\
\hline $6 \cdot 0$ & $66 \cdot 0$ & 27.8 & 253 & 245 \\
\hline $11 \cdot 0$ & $66 \cdot 6$ & 27.9 & 256 & 246 \\
\hline 15.0 & $66 \cdot 8$ & $28 \cdot 1$ & 257 & 249 \\
\hline $33 \cdot 0$ & $67 \cdot 0$ & 28.6 & 258 & 255 \\
\hline $60 \cdot 0$ & 66.8 & $31 \cdot 0$ & 257 & 285 \\
\hline 120.0 & $64 \cdot 1$ & 29.6 & 243 & 267 \\
\hline \multirow{2}{*}{\multicolumn{5}{|c|}{ Modified waxy maize }} \\
\hline & & & & \\
\hline 0.0 & $16 \cdot 3$ & $5 \cdot 2$ & NA & NA \\
\hline 0.5 & 37.6 & $13 \cdot 1$ & 131 & 153 \\
\hline 1.0 & 37.9 & $13 \cdot 3$ & 133 & 157 \\
\hline $10 \cdot 0$ & 38.8 & 13.8 & 138 & 166 \\
\hline 30.0 & 39.8 & 14.3 & 144 & 176 \\
\hline
\end{tabular}

a $\Delta D(\%)=\left(\frac{D-D_{0}}{D_{0}}\right) \times 100 ; D$ is the average diameter after heating for a time $t$, and $D_{0}$ is diameter before heating.

${ }^{\mathrm{b}} \Delta \sigma(\%)=\left(\frac{\sigma-\sigma_{0}}{\sigma_{0}}\right) \times 100 ; \sigma$ is the standard deviation of the granules after heating for a time $t$, and $\delta_{0}$ is the standard deviation before heating.

NA: not applicable.

shear rate $\left(\dot{\gamma}_{\mathrm{c}}\right)$ is considered to be an important quantity in polydisperse dispersions of rigid spheres exhibiting shear thickening (Barnes, 1989; Boersma et al., 1990, 1991; Laun et al., 1991).

For each cowpea starch dispersion that exhibited shear thickening behavior, the value of $\dot{\gamma}_{c}$ was determined as the point of intersection of the regression lines of the two data segments of a $\log -\log$ plot of shear stress vs. shear rate

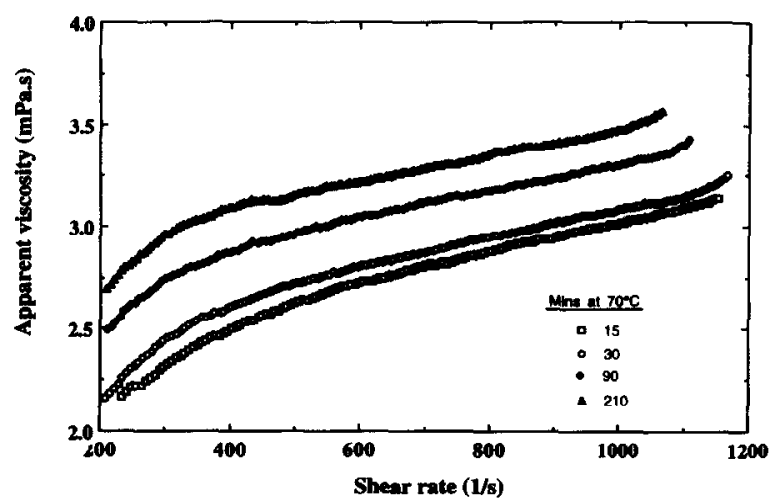

Fig. 4. Shear thickening behavior of $2.6 \%$ corn starch dispersions heated at $70^{\circ} \mathrm{C}$.
(Okechukwu and Rao, 1996). In the range $10 \mathrm{~s}^{-1}$ to $\dot{\gamma}_{\mathrm{c}}$, the dispersions exhibited shear thinning $(n<1.0)$ while in the range $\dot{\gamma}_{\mathrm{c}}$ to $900 \mathrm{~s}^{-1}$ they exhibited dilatant behavior. Also, $\dot{\gamma}_{c}$ gradually increased with time of heating at $67^{\circ} \mathrm{C}$ apparently in response to the increasing granule mean diameter and GSID. The shift of $\dot{\gamma}_{c}$ to higher shear rates as GSID increases is consistent with observations on dispersions of rigid spheres (Barnes, 1989; Boersma et al.,

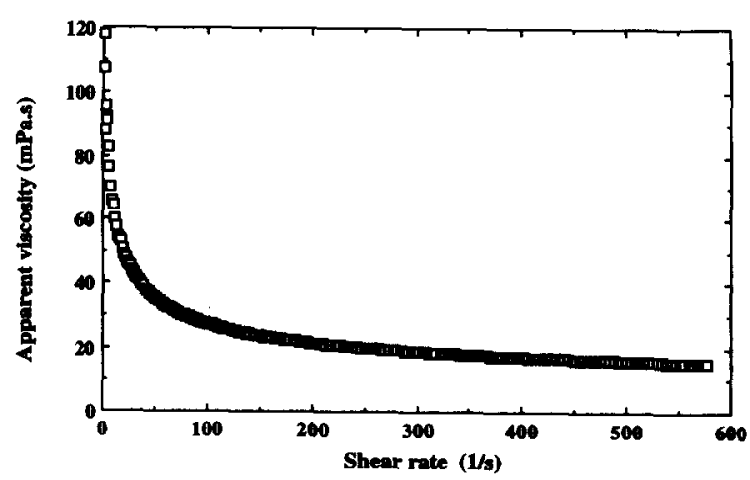

Fig. 5. Shear thinning behavior of $2.6 \%$ corn starch dispersions heated at $85^{\circ} \mathrm{C}$. 


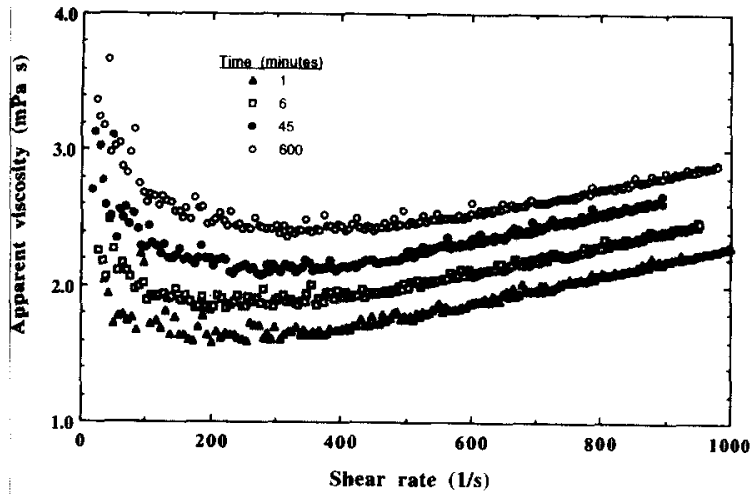

Fig. 6. Apparent viscosity behavior of $2.6 \%$ cowpea starch dispersions heated at $67^{\circ} \mathrm{C}$. Note the initial shear thinning and initiation of shear thickening at a critical shear rate

1990) (Table 2). Values of $\dot{\gamma}_{c}\left(337-372 \mathrm{~s}^{-1}\right)$ over the granule mass fraction range $0.20-0.25$ are higher than those $\left(10-50 \mathrm{~s}^{-1}\right)$ found in Bagley and Christianson (1982) and Christianson and Bagley (1984) at granule mass fractions $0.6-0.7$, but are in qualitative agreement with results for rigid spheres where $\dot{\gamma}_{c}$ decreases with increase in granule mass fraction. However, $\dot{\gamma}_{c}$ increased with the mean granule diameter and the dispersed phase volume fraction. At first this observation would appear to be inconsistent with observations on dispersions of spherical rigid solid particles of narrow size distribution where $\dot{\gamma}_{c}$ decreased with increase in volume fraction and particle size (Barnes, 1989; Boersma et al., 1990). However, in the studied starch dispersions, an increase in the granule mass fraction was always accompanied by a concomitant increase in granule diameter and GSID. Therefore, it appears that in the studied starch dispersions, the GSID was a more dominant factor than volume fraction and mean granule size in influencing whether $\dot{\gamma}_{\mathrm{c}}$ increased or decreased.

\section{Influence of GSID on flow behavior}

Further insight into the variation of the flow behavior index, $n$, can be gained by examining its relationship with GSID as granule swelling progressed. Figure 7 shows that the shear thickening $(n>1.0)$ shown by corn and cowpea starch dispersions in the early stages of granule growth was associated with a low standard deviation of the GSID. As gelatinization progressed, GSID broadened with a concomitant decrease in the magnitude of the flow behavior index. This decrease in the severity of shear thickening with increase in standard deviation of the particle size during the early stages of starch granule swelling is consistent with the observation of Barnes (1989).

Increase in granule size standard deviation resulted in decrease in the severity of shear thickening and increase in the pseudoplasticity of the starch dispersion. Over the temperature range $70-90^{\circ} \mathrm{C}$, the variation of $n$ with the standard deviation, SD, of the corn starch GSID was linear (eqn (2)) with an $R^{2}$ of 0.98 :

$$
n=2.17-0.0968 \times \mathrm{SD} \text {. }
$$

An interesting result from eqn (2) is that the maximum value of the flow behavior index of aqueous corn starch dispersion would be about 2.2. However, because of the empirical nature of the equation, higher values may be attained.

Decrease in the severity of shear thickening may be attributed to a number of factors including polydispersity in granule size, granule volume fraction, and deformability of the dispersed granules. Over a wide range of swelling of cowpea starch granules heated at $67-80^{\circ} \mathrm{C}, n$ decreased from 1.3 to 0.56 (Fig. 7) with increase in $\left(\mathrm{SD} / \mathrm{SD}_{0}\right)$ of unruptured granules in the starch dispersion, where $\mathrm{SD}_{0}$ is the standard deviation of the unheated granules. Over a granule SD of $15-32 \mu \mathrm{m}$, a power law equation correlated the two dimensionless variables $n$ and (SD/ $\left.\mathrm{SD}_{0}\right)$ :

$$
n=2 \cdot 36\left[\frac{\mathrm{SD}}{\mathrm{SD}_{0}}\right]^{-1 \cdot 4} R^{2}=0 \cdot 95
$$

Therefore, in addition to the influence of the concentration of starch on the magnitude of $n$ (Colas, 1986; Evans and Haisman, 1979), increase in polydispersity of the gelatinized granules appears to be an important factor in decreasing the severity of shear

Table 2. Critical shear rates and power law parameters for shear thinningand shear thickening segments shown by cowpea starch held

\begin{tabular}{|c|c|c|c|c|c|c|c|}
\hline \multirow{2}{*}{$\begin{array}{l}\text { Heating } \\
\text { time(min) }\end{array}$} & \multirow{2}{*}{$\begin{array}{l}\text { Critical shear } \\
\text { rate }\left(\mathrm{s}^{-1}\right)\end{array}$} & \multicolumn{3}{|c|}{ Shear thinning range } & \multicolumn{3}{|c|}{ Shear thickening range } \\
\hline & & $K\left(\mathrm{mPa} \mathrm{s}^{n}\right)$ & $n$ & $R^{2}$ & $K\left(\mathrm{mPa} \mathrm{s}^{\prime \prime}\right)$ & $n$ & $R^{2}$ \\
\hline 0.5 & 337 & 2.45 & 0.94 & 0.997 & 0.27 & 1.31 & 0.998 \\
\hline 1.0 & 340 & 3.44 & 0.91 & 0.994 & 0.36 & 1.32 & 0.999 \\
\hline 4.0 & 343 & 3.75 & 0.87 & 0.976 & 0.30 & 1.30 & 0.999 \\
\hline $\begin{array}{l}+\cdot 0 \\
6 \cdot 0\end{array}$ & 350 & 3.25 & 0.89 & 0.996 & 0.30 & $1 \cdot 30$ & 0.999 \\
\hline $15 \cdot 0$ & 354 & 3.09 & 0.89 & 0.991 & 0.30 & 1.28 & 0.998 \\
\hline 45.0 & 365 & 4.40 & 0.87 & 0.994 & 0.41 & 1.26 & 0.998 \\
\hline 68.0 & 367 & $4 \cdot 60$ & 0.85 & 0.990 & 0.43 & 1.26 & 0.999 \\
\hline $90 \cdot 0$ & 372 & 3.44 & 0.91 & 0.996 & 0.43 & 1.26 & 0.999 \\
\hline
\end{tabular}
at $67^{\circ} \mathrm{C}$ 


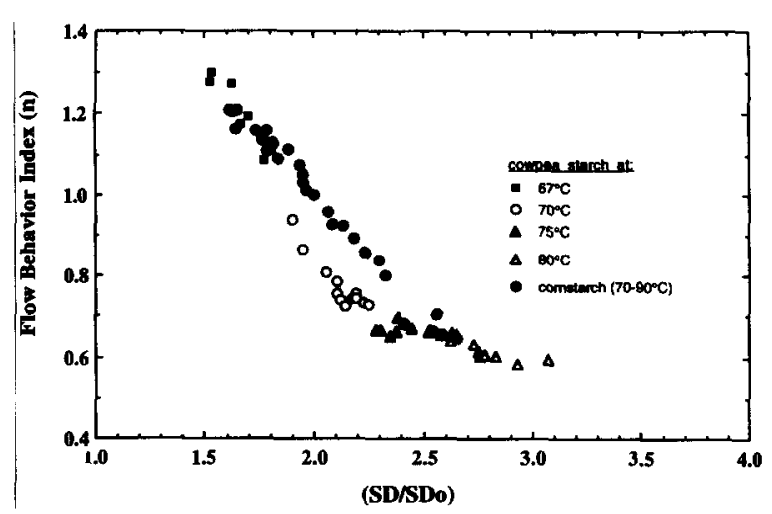

Fig. 7. Relationship between flow behavior index $(n)$ and ratio of standard deviation of starch granule size distribution $\left(\mathrm{SD} / \mathrm{SD}_{0}\right) ; \mathrm{SD}_{0}$ is the standard deviation of ungelatinized starch granules.

thickening and increasing pseudoplasticity of gelatinized starch dispersions.

\section{Starch volume fraction, granule size, and viscosity}

Increase in the volume fraction of solids in a starch dispersion can be achieved by either increasing the concentration of the solids or through volume expansion of the solids. The relationship between granule swelling and the notional volume fraction $(c Q)$ was explored in heated dispersions of corn and cowpea starches. For dimensional consistency with $c Q$, the former was expressed as $\left(D / D_{0}\right)^{3}$, where $D$ and $D_{0}$ are the diameters of gelatinized and ungelatinized starch granules, respectively. Slightly different linear relationships were found for corn and cowpea starch dispersions, eqns (4) and (5), respectively, but the scatter in the data points and the modest values of $R^{2}$ $(0.86$ and 0.9$)$ reflect the difficulty and subjectivity involved in siphoning off the supernatant liquid after centrifugation when determining $c Q$.

$$
\begin{aligned}
& c Q=0.156+0.0109\left(\frac{D}{D_{0}}\right) \\
& c Q=0.0828+0.0133\left(\frac{D}{D_{0}}\right) .
\end{aligned}
$$

The Krieger-Dougherty relationship (eqn (6)) is theoretically well founded and contains two parameters, $[\eta]$ and $\phi_{\mathrm{m}}$.

$$
\eta_{\mathrm{r}}=\left(1-\frac{\phi}{\phi_{\mathrm{m}}}\right)^{-|\eta| \phi_{\mathrm{m}}} \text {. }
$$

Theoretically, $[\eta]$ should be 2.5 for rigid spheres and $\phi_{\mathrm{m}}$ should be about 0.62 if the spheres are of uniform diameter (Krieger, 1985), but Choi and Krieger (1986) found it necessary to use values of $[\eta]$ of $2.65-3.19$ to fit eqn (6) to viscosity-volume fraction data on sterically stabilized polymethylmethacrylate spheres. In addition, because of the polydisperse and deformable nature of gelatinized starch dispersions, it is not surprising that attempts to predict their viscosity with eqn (6) (Noel et al., 1993; Ellis et al., 1989) were not successful.

The ratio of mean granule diameters $\left(d=D / D_{0}\right)$ was used to correlate consistency index data on corn and cowpea starch dispersions (Fig. 8). Initially, $K$ increased gradually and later very rapidly as granule mean diameter approached the highest value (3.5) of mean diameter ratio (d). At low values of $c Q$, the granules were sufficiently apart and did not affect dispersion viscosity substantially. As $c Q$ increased above a threshold value, granule-granule interaction increased accompanied by a large increase in dispersion viscosity. An exponential function (eqn (6)) satisfactorily described the dependency of $K$ on $d$ over the mean granule size range $30-65 \mu \mathrm{m}$.

$$
K=K_{\mathrm{o}} \exp (\alpha d) \quad R^{2}=0.96
$$

where $K_{\mathrm{o}}$ and $\alpha$ are empirical parameters with estimates of $2.40 \times 10^{-5}\left(\mathrm{mPa} \mathrm{s}^{\mathrm{n}}\right)$ and 4.84 , respectively. The maximum mean diameter ratio $\left(d_{\max }\right)$ of 3.5 corresponded to a dispersed phase volume fraction of $65 \%$. A single equation for data of corn and cowpea starches is noteworthy. Cornstarch approached a limiting mean diameter ratio of 3.3 at a dispersed phase volume fraction of $55 \%$. The difference in values of $d_{\max }$ may be attributed to differences in granule shape and the ability of the granules to expand.

\section{Effect of granule size on casson yield stress}

The yield stress of cowpea starch dispersions was estimated using the Casson equation:

$$
\alpha^{0.5}=K_{0 \mathrm{C}}+K_{\mathrm{C}} \dot{\gamma}^{0.5}
$$

where the Casson yield stress is $\sigma_{0 \mathrm{C}}=\left(K_{0 \mathrm{C}}\right)^{2}$ and the Casson viscosity is $\eta_{\mathrm{C}}=\left(K_{\mathrm{C}}\right)^{2}$. In agreement with the findings of Christianson and Bagley (1984), the yield

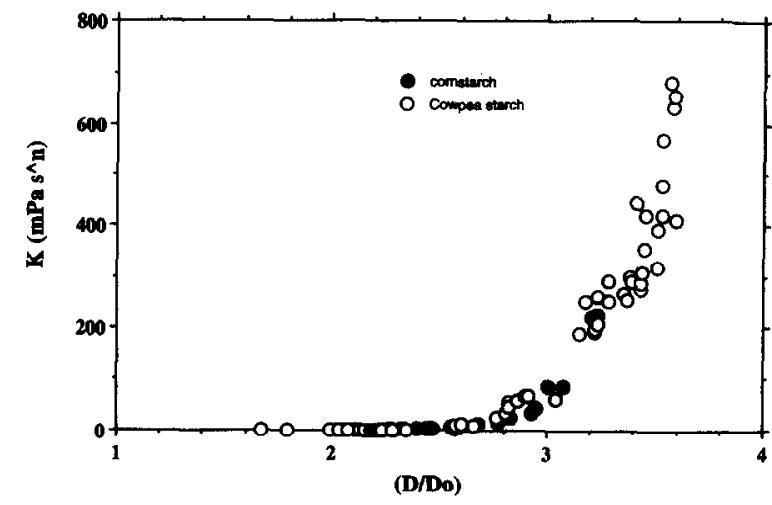

Fig. 8. Consistency index $(K)$ of corn and cowpea starch dispersions as function of diameter ratio $\left(D / D_{0}\right) ; D_{0}$ is the mean diameter of ungelatinized starch granules. 
stress of gelatinized starch dispersions increased strongly with the extent of granule swelling and approached a limiting value at a mean diameter ratio $\left(D / D_{0}\right)$ of 3.6. Appreciable values of yield stress were obtained above mean diameter ratios of 2.8 , corresponding to a sediment volume fraction of 0.4 that was higher than the minimum granule mass fraction for shear thickening. The Casson viscosity $\eta_{\mathrm{C}}$ also increased with granule swell within the narrow range of $0.03-0.22$ (Pa s) for dispersions that exhibited shear thinning (Fig. 9); it is useful because it is equivalent to the infinite shear viscosity of shear thinning dispersions (Metz et al., 1979). An exponential equation described the relationship between $\sigma_{0 C}$ and $\left(D / D_{0}\right)$ and a linear relationship described that between $\eta_{\mathrm{C}}$ and $\left(D / D_{0}\right)$ (Fig. 9).

\section{Time-dependent behavior of modified waxy maize starch dispersions}

Time-dependent shear thinning, commonly known as thixotropic, rheological behavior has been extensively studied (Mewis, 1979). On the other hand, antithixotropic rheological behavior has not been studied extensively. The apparent viscosity, at constant shear rates between 100 and $400 \mathrm{~s}^{-1}$, versus time data for a $5 \%$ gelatinized modified waxy maize starch
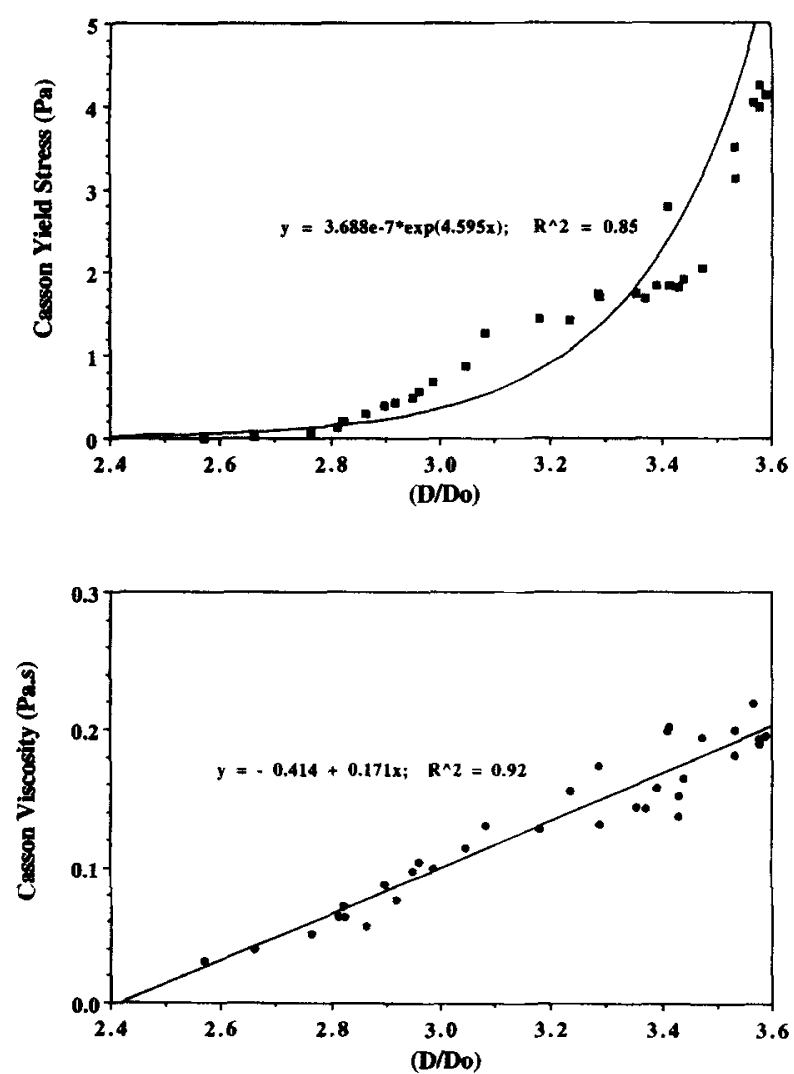

Fig. 9. Yield stress and Casson viscosity of cowpea starch dispersions.

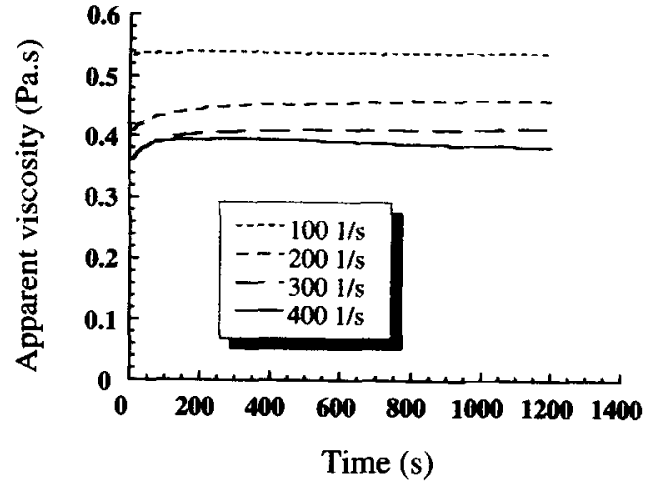

Fig. 10. Antithixotropic behavior of $5 \%$ modified waxy maize starch dispersion that is evident at a shear rate of $200 \mathrm{~s}^{-1}$.

dispersion are shown in Fig. 10. Increasing shear rates resulted in increase in the antithixotropic behavior of the dispersion; antithixotropic behavior was negligible at $100 \mathrm{~s}^{-1}$ but clearly evident at $200 \mathrm{~s}^{-1}$. At high shear rates $\left(400 \mathrm{~s}^{-1}\right)$ after the initial apparent viscosity increase a small gradual decrease was observed, which may be due to the centrifugal forces inside the gap that forced some of the sample out. The starch concentration also had a positive effect on the magnitude of the antithixotropic hehavior. The Weltmann model (eqn (9)) (Weltmann, 1943) described well the time-dependent data as summarized in Table 3:

$$
\sigma=A-B \log t
$$

where $\sigma$ is shear stress (Pa), $t$ is time, and $A$ and $B$ are constants; in antithixotropic behavior $B$ takes positive values. Data at $300 \mathrm{~s}^{-1}(3$ and $4 \%)$ and $50 \mathrm{~s}^{-1}(5 \%)$ are not shown because they could not be consistently reproduced.

\section{Viscoelastic behavior of starch dispersions}

Viscoelastic properties of starch dispersions are relevant to our understanding of the structure of such dispersions (Biliaderis, 1992) and their flow behavior (Boersma et al., 1992; Chow and Zukoski, 1995b). Figure 11 shows the dynamic properties, storage modulus $\left(G^{\prime}, \mathrm{Pa}\right)$ and loss modulus $\left(G^{\prime \prime}, \mathrm{Pa}\right)$ vs. frequency $\left(\mathrm{rad} \mathrm{s}^{-1}\right)$ of gelatinized MWM starch dispersions of 3, 4 and $5 \%$ solids. In all dispersions, $G^{\prime}$ was much higher than $G^{\prime \prime}$, i.e. the dispersions exhibited gel-like behavior over the studied range of frequencies. Both $G^{\prime}$ and $G^{\prime \prime}$ increased with concentration and showed a weak dependence on frequency at low values of this variable. This dependency was more pronounced in dispersions of higher concentration.

The Cox-Merz rule (eqn (10)) was not applicable to gelatinized MWM starch dispersions in the range of concentrations studied, in that the complex viscosity $\left(\eta^{*}\right)$ was higher than the flow viscosity over the range of frequencies and shear rates studied (Fig. 12). 
Table 3. Weltman equation parameters for heated modified waxy maize starch dispersions

\begin{tabular}{cccccc}
\hline \multirow{2}{*}{$\begin{array}{c}\text { Weltman } \\
\text { parameter }\end{array}$} & Conc. (\%) & \multicolumn{3}{c}{ Shear rate $\left(\mathrm{s}^{-1}\right)$} \\
\cline { 3 - 5 } & & 50 & 100 & 200 & 300 \\
\hline \multirow{2}{*}{ A } & 3 & $6.97 \times 10^{-2}$ & $5.76 \times 10^{-2}$ & $4.22 \times 10^{-2}$ & \\
& 4 & $2.97 \times 10^{-1}$ & $2.08 \times 10^{-1}$ & $1.72 \times 10^{-1}$ & $9.54 \times 10^{-2}$ \\
& 5 & $5.39 \times 10^{-1}$ & $3.88 \times 10^{-1}$ & $3.55 \times 10^{-1}$ & \\
B & 3 & $1.58 \times 10^{-3}$ & $5.71 \times 10^{-4}$ & $1.66 \times 10^{-5}$ & $7.83 \times 10^{-3}$ \\
& 4 & $3.15 \times 10^{-3}$ & $2.07 \times 10^{-3}$ & $5.29 \times 10^{-3}$ & $8.79 \times 10^{-3}$ \\
\hline
\end{tabular}

${ }^{a}$ Magnitudes of correlation coefficient $(R)$ were 0.999 .

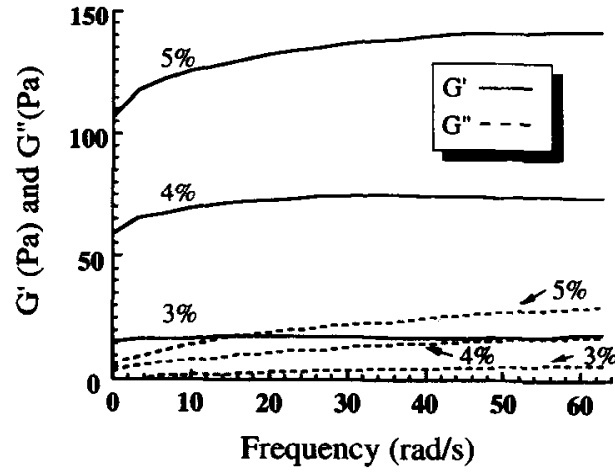

Fig. 11. Storage $\left(G^{\prime}\right)$ and loss $\left(G^{\prime \prime}\right)$ moduli of $3 \%, 4 \%$, and $5 \%$ solids modified waxy maize starch dispersions as a function of oscillatory frequency; note that $G^{\prime}$ is much higher than $G^{\prime \prime}$, which is a characteristic of true gels.

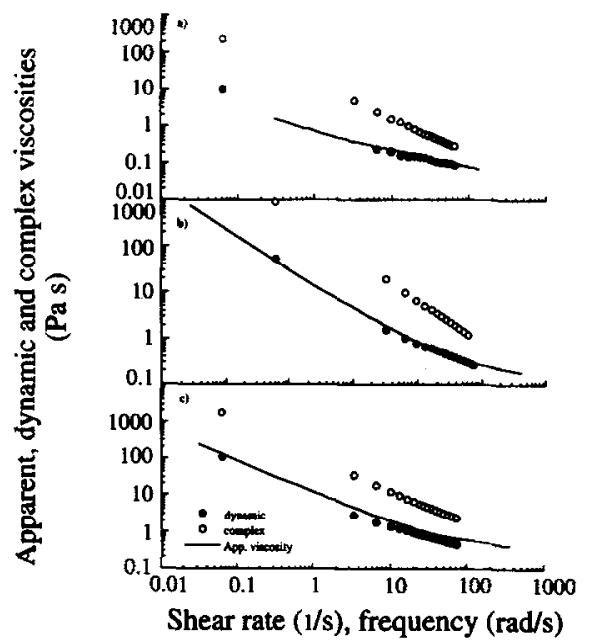

Fig. 12. Cox-Merz plots for 3, 4, and 5\% modified waxy maize starch dispersions. Because complex viscosity $\left(\eta^{*}\right)$ was not equal to apparent viscosity, the dispersions behaved as true gels.

$$
\eta^{*}(\omega)=\left.\eta(\dot{\gamma})\right|_{\omega=\dot{\gamma}}
$$

where $\eta^{*}=\left(G^{*} / \omega\right), \omega$ is the frequency of oscillation $\left(\mathrm{rad} \mathrm{s}^{-1}\right)$ and $G^{*}=\sqrt{\left.\left(G^{\prime}\right)^{2}+G^{\prime \prime}\right)^{2}}$. Deviation from the Cox-Merz rule also reflects the gel-like nature of the gelatinized dispersions (Ross-Murphy, 1984). The complex viscosity can be further expressed in terms of real and imaginary parts, $\eta^{*}=\eta-\mathrm{i} \eta^{\prime \prime}$; it is interesting to note that the dynamic viscosity $\left(\eta^{\prime}\right)$ is in better agreement with the apparent viscosity than is $\eta^{*}$ (Fig. 12).

Evans and Lips (1992) extended the work of Buscall et al. $(1982 \mathrm{a}, \mathrm{b})$ to relate the shear modulus, $G$, of a dispersion of spherical particles to various characteristics of the particles and the continuous medium:

$$
\begin{aligned}
G= & \frac{\phi_{c p} n G_{\mathrm{p}}}{5 \pi\left(1-\mu_{\mathrm{p}}\right)} \\
& {\left[\phi_{\text {red }}^{1 / 3}\left(1-\phi_{\text {red }}^{-1 / 3}\right)^{1 / 2}-\frac{8}{3} \phi_{\text {red }}^{2 / 3}\left(1-\phi_{\text {red }}^{-1 / 3}\right)^{3 / 2}\right] }
\end{aligned}
$$

where $G_{\mathrm{p}}$ is the shear modulus of the material of the spherical particle, $\mu_{\mathrm{p}}$ is Poisson's ratio, $\phi_{\mathrm{cp}}$ is the volume fraction for close packing and $\phi_{\text {red }}$ is the reduced phase volume fraction $\left(\phi / \phi_{\mathrm{cp}}\right)$ given by $\phi_{\text {red }}=\left(a_{0} / h\right)^{3} ; a$ is the undeformed particle radius, and $h$ is the distance to the nearest particle. Evans and Lips suggested that for fully swelled starch granules, the phase volume fraction $\phi=c Q$. However, agreement between experimental data on starch dispersions and theory was poor but the theoretical trends were followed by Sephadex spheres used in chromatography columns (Evans and Lips, 1992, 1993). The main reasons for the difference between theory and experiment for heated starch dispersions were that the granules were not truly spherical and rigid ('macroscopic elastic spheres') and that they were polydisperse in nature. Also, it was not possible to determine the representative viscoelastic behavior of all the granules from the stress-strain data on a few granules using the cell poker technique (McConnaughey and Petersen, 1980) that still provides mainly qualitative results (Zahalak et al., 1990). Plots of SGM fraction vs. normalized (based on the value of $G^{\prime}$ at $c Q=1$ ) $G^{\prime}$ data on starches resulted in three groups: chemically modified, root and tuber, and cereal (Evans and Lips, 1992) that were attributed to 'structural origins' of the starches. 


\section{CONCLUSIONS}

The GSID and mean diameter are important properties of the dispersed phase which exert considerable influence on the viscosity of gelatinized dispersions of unruptured corn and cowpea starch granules. The flow behavior index $n$ showed a strong dependency on SD and provided an analytical route for the transition of flow behavior from shear thickening at the early stages of granule swelling to subsequent shear thinning. SD increased with granule swelling and appeared to be a dominant variable for predicting the $\dot{\gamma}_{c}$ for the onset of shear thickening in starch dispersions. Shear thickening decreased while pseudoplasticity increased with increase in granule SD as granule swelling progressed. The increase in the consistency index $(K)$ during granule swelling correlated well with increase in the granule mean diameter. High values of $K$ were attained as the granules swelled to about 3.5 times the mean diameter of the raw starch granules and at a dispersed phase volume fraction close to the maximum volumetric loading $(60 \%)$ for monodisperse spheres. Dispersions of modified waxy maize starch exhibited antithixotropic rheological behavior and gel-like behavior, i.e. values of $G^{\prime}$ much greater than $G^{\prime \prime}$; they did not follow the Cox-Merz rule, reflecting their gellike behavior.

\section{ACKNOWLEDGEMENTS}

The authors are grateful for financial support from USDA NRI Research Project, and JNICT, Lisbon, Portugal.

\section{REFERENCES}

Doublier, J.L. (1981) Starch/Stärke 33, 415-420.

Colas, B. (1986) Lebinsnuttell Wissenschaft und Technologie 19, 308-311

Bagley, E.B. and Christianson, D.D. (1982) J. Texture Studies 13, 115-126.

Barnes, H.A. (1989) J. Rheol. 33, 329-366.

Biliaderis, C. G. (1992) In Developments in Carbohydrate Chemistry, ed. R. J. Alexander and H. F. Zobel, pp. 87135. AACC, St Paul, MN.

Boersma, W.H., Laven, J. and Stein, H.N. (1990) Am. Inst. Chemical Eng. J. 36, 321-322..

Boersma, W.H., Baets, P.J.M., Laven, J. and Stein, H.N. (1991) J. Rheol. 35, 1093-1120.

Boersma, W.H., Laven, J. and Stein, H.N. (1992) J. Colloid and Interface Sci. 149, 10-22.

Buscall, R., Goodwin, J.W., Hawkins, M.W. and Ottewell, R.H. (1982) .J. Chem. Soc. Faraday Trans. 78, 2873-2887.

Buscall, R., Goodwin, J.W., Hawkins, M.W. and Ottewell, R.H. (1982) J. Chem. Soc. Faraday Trans. 78, 2889-2899.

Cheng-yi, L. and Shin-ming, C. (1981) J. Food Sci. 46, 78-82.

Choi, G.R. and Krieger, I.M. (1986) J. Colloid and Interface Sci. 113, 101-113.
Chow, M.K. and Zukoski, C.F. (1995) J. Rheol. 39, 15-32.

Chow. M.K. and Zukoski, C.F. (1995) J. Rheol. 39, 33-59.

Christianson, D.D. and Bagley, E.B. (1984) Cereal Chem. 61, 500-503.

D'Haene, P., Mewis, J. and Fuller, G.G. (1993) J. Colloid and Interface Sci. 156, 350-358.

Dail, R.V. and Steffe, J.F. (1990) J. Food Sci. 55, 1764 1765.

Dail, R.V. and Steffe, J.F. (1990) J. Food Sci. 55, 16601665.

Dintzis, F.R. and Bagley, E.B. (1995) J. Appl. Polymer Sci. 56, $637-640$.

Doublier, J.L. (1987) J. Cereal Sci. 5, 247-262.

Einstein, A. (1906) Ann. Physik 19, 289-306.

Einstein. A. (1911) Ann. Physik 34, 591-592.

Eliasson, A.C. (1986) J. Texture Studies 17, 253-265.

Ellis, H.S., Ring, S.G. and Whittam, M.A. (1989) J. Cereal Sci. 10, 33-44.

Evans, I.D. and Haisman. D.R. (1979) J. Texture Studies 10, 347-370.

Evans, I.D. and Lips, A. (1992) J. Texture Studies 23, 69-86.

Evans, I. D. and Lips, A. (1993) In Food Colloids and Polymers: Stability and Mechanical Properties, ed. E. Dickinson and P. Walstra, pp. 214-223. The Royal Society of Chemistry, Cambridge, UK.

Galliard, T., Bowler, P. (1987) In Starch: Properties and Potential, Critical Reports on Applied Chemistry, ed. T. Galliard, Vol. 13, pp. 54-78. John Wiley and Sons, New York.

Giboreau, A., Cuvelier, G. and Launay, B. (1994) J. Texture Studies 25, 119-137.

Harrod, M. (1989) J. Food Process Eng. 11, 257-275.

Hoffmann, R.L. (1972) Trans. Soc. Rheol. 16, 155-173.

Jinescu, V.V. (1974) International Chemical Eng. 14, 397-420.

Ketz, R.J., Prud'homme, R.K. and Graessley, W.W. (1988) Rheol. Acta 27, 531-539.

Kokini, J.L., Lai, L.-S. and Chedid, L.L. (1992) Food Technol. 466, 124-139.

Krieger, I. J. (1985) In Polymer Colloids, ed. R. Buscall, T, Corner and J. F. Stageman, pp. 219-246. Elsevier Applied Science, New York.

Laun, H.M., Bung, R. and Schmidt, F. (1991) J. Rheol. 35, 999-1034.

Leach, H.W., McGowen, L.D. and Schoch, T.J. (1959) Cereal Chem. 36, 535-545.

Liu, H. and Lelievre, J. (1993) Carbohydrate Polymers 20, 1-5. Lund, D. (1984) Critical Reviews in Food Sci. and Nutrition 20, 249-273.

McConnaughey, W.B. and Petersen, N.O. (1980) Rev. Sci. Instrum. 51, 575-580.

Metz, B., Kossen, N. W. F. and van Suijdam, J. C. (1979) In Advances in Biochemical Engineering, ed. T. K. Ghose, A. Fiechter and N. Blakebrough, Vol. 2, pp. 103-156. Springer Verlag, New York.

Metzner, A.B. (1985) J. Rheol. 29, 739-775.

Mewis, J. (1979) J. Non-Newtonian Fluid Mech. 6, 1-20.

Nocl, T. R.., Ring, S. G. and Whittam, M. A. (1993) In Food Colloids and Polymers: Stability and Mechanical Properties, ed. E. Dickinson and P. Walstra, pp. 126-137. Royal Society of Chemistry, Cambridge, UK.

Okechukwu, P.E. and Rao, M.A. (1995) J. Texture Studies 26, 501-516.

Okechukwu, P.E. and Rao, M.A. (1996) J. Texture Studies 27, 159-173.

Rockland, L.B., Jones, F.T. and Hahn, D.M. (1977) J. Food Sci. 42, 1204-1210.

Ross-Murphy, S. B. (1984) In Biophysical Methods in Food Research, ed. H. W.-S. Chan, pp. 138-199. Blackwell Scientific, London. 
Colloidal Dispersions. Cambridge University Press, Cambridge, UK.

Schoch, T.J.Maywald, E.C. (1968) Cereal Chem. 45, 564-568.

Svegmark, K. and Hermansson, A.M. (1992) Food Structures 12, 181-193.

Weltmann, R.N. (1943) J. Applied Phys. 14, 343-350.

Wong, R.B.K., Lelievre, J. (1982) J. Applied Polymer Sci. 27,
1433-1440.

Zahalak, G.I.McConnaughey, W.B.Elson, E.L. (1990) J. Biomechanical Eng. 112, 283-294.

Ziegler, G.R., Thompson, D.R., Casanovas, J. (1993) Cereal Chem. 70, 247-251. 